\title{
Independent DTNs Message Deletion Mechanism for Multi-copy Routing Scheme
}

\author{
Sathita Kaveevivitchai \\ The University of Tokyo \\ 7-3-1 Hongo Bunkyo-ku \\ Tokyo 113-8656 JAPAN \\ wan@hongo.wide.ad.jp
}

\author{
Hiroshi Esaki \\ The University of Tokyo \\ 7-3-1 Hongo Bunkyo-ku \\ Tokyo 113-8656 JAPAN \\ hiroshi@wide.ad.jp
}

\begin{abstract}
In this paper we discuss the independent message deletion mechanism for Delay Tolerant Networks (DTNs) multi-copy routing schemes, and the enhancement of this method with the knowledge of human mobility patterns. This mechanism can optimize the resource utilization and improve message delivery performance. In DTNs, communication is achieved by the movement of mobile devices and the ability to store-carry-and-forward the messages. Since contacts in this network occur opportunistically, multi-copy routing scheme suggests messages to be replicated, carried and forwarded by several devices to enhance the delivery ratio though it consumes greater resources compared to singlecopy routing scheme. We propose the method to optimize the resource utilization in multi-copy routing scheme by efficiently deleting the copy of delivered message. This method reduces storage consumption, and avoids devices from wasting their bandwidth, and energy, hence, improves the performance of DTNs message delivery in multi-copy routing scheme. We have taken the intrinsic characteristic of human movements, known as, "Human Gathering Points" to enhance our proposed method. We evaluate the proposed method in the simulations with real human movement data trace on three major DTNs multi-copy routing schemes, Epidemic, Spray and Wait, and ProPHET. The result shows significant improvement in message delivery (10-50\%) while reduces the resource consumption (overhead) by $30-70 \%$. Its independent characteristic makes it possible to be applied with any multi-copy routing protocol though the degree of impact is varied based on the underlying routing scheme.
\end{abstract}

\section{Categories and Subject Descriptors}

C.2.1 [Network Architecture and Design]: Wireless Communication; C.2.2 [Network Protocols]: Resource Management Protocol-performance measures

\section{General Terms}

Algorithms, Performance, Theory

\author{
Keywords \\ Delay Tolerant Networks, Human Mobility Patterns, Mes- \\ sage Deletion Mechanism
}

\section{INTRODUCTION}

As we move from the Information era to Attention era, where information can be generated and shared anytime, anywhere and by anyone, there is a need for individuals to create and consume information freely and instantly. Therefore it demands the constant communication on mobile personal devices. Through the movement of these devices, the connection and message delivery become possible in sparsely connected networks known as Delay Tolerant Networks (DTNs) [1].

The concept of DTNs has addressed the network in challenging environments. The movement of mobile devices create frequent topology changes, disruptions, and network partitions. Moreover, the limited storage and energy of these devices also cause the intermittent connectivity and the absence of end-to-end connectivity. To overcome these challenges, one needs to provide the protocols that can tolerate the dynamic network environment with abrupt changes and high delay while stay tune to the optimization of resources utilization.

To achieve messages delivery upon above requirements, many DTNs routing algorithms had been proposed in recent years, which can be categorized into two main classes: single-copy routing scheme[2] and multi-copy routing scheme[3, 4, 5]. While the multi-copy routing scheme promises higher delivery ratio, there are more resources to be consumed. The method to achieve high delivery performance at the minimal cost is still to be devised. One way to enhance the performance of DTN message delivery is through message management scheme such as buffer management and message deletion mechanism. We need to optimize the resources usage (storage, bandwidth and energy) while maintaining high delivery ratio as in multi-copy routing scheme.

Generally, the storage managements in DTNs are often integrated with the routing algorithm such as Hop-based TTL in Spray and Wait[4], or passive-cure in Potential-based Entropy Adaptive Routing (PEAR)[17]. So far, there are several works regarding buffer management policies which can work independently. Yet our method differs from those since it works as preventive measure for resources exhaustion and 
storage overflow problems rather than counter measure as in $[20,21]$. The contribution of this paper is the proposal of independent message deletion mechanism for DTNs multicopy routing scheme. And the proposal of its enhancement with the "Human Gathering Points", the locations where people naturally gather together, detailed explanation in Section 2.2. The key design of our protocol is to cut-off unnecessary resource usage through the deletion of the copies/replicas of delivered messages. It will prevent nodes from the storage overflow problems, reduces storage and energy consumption, avoid unnecessary transfer of useless message replicas, therefore it can enhance the performance of DTNs message delivery. We evaluate our proposed method by simulation with the real human movement data traces[12], on three major DTNs routing algorithms: Epidemic[3], Spray and Wait[4], and ProPHET[5].

The results show that our method improves the performance of multi-copy routing algorithms though the degree of impact is different based on the underlying routing algorithm. The remainder of this paper is organized as follows. Section 2 explains our proposed deletion mechanism, mobility patterns and "Human Gathering Points". Section 3 describes the simulation environments and presents the results. Related work is presented in Section 4. Finally we conclude in Section 5 and present our future work.

\section{DELETION MECHANISM AND MOBILITY PATTERNS}

Oftentimes, the countless replica in multi-copy routing scheme, overwhelm the limited network resources. When the messages had reached their destinations, their replicas become useless though they are still occupying storage space and being forwarded to other nodes. Without the proper resource management scheme, these useless replicas can cause the degradation of the DTNs delivery performance. We propose a message deletion scheme, which will be able to apply independently from the underlying routing protocols.

Since the devices in DTNs environment are generally carried by human, their movements are closely correlated to the human movements. The key element in our design is the study and application of the heuristic knowledge of actual human mobility. This method is proven to be efficient in terms of: (i) Ability to remove copies of delivered messages in timely manner; (ii) improvement in resources utilization (bandwidth, storage).

We use the acknowledgment from the destination node to indicate that the message has already been received and its replicas can be deleted from the intermediate nodes. We separate our design into two parts: (i) methods for distributing acknowledgment messages (ACK); (ii) The use of Helper nodes at Human Gathering Points to relay ACK messages.

\subsection{ACK Distribution Method to Delete Replicas}

The ACK messages can be distributed in two different ways, either passive or active policies, as it is illustrated in Figure. 1. In passive distribution policy, ACK of any message $\mathrm{m}$ will not be expressed to any encountering node (B) unless the encountering node makes an attempt to send a copy of

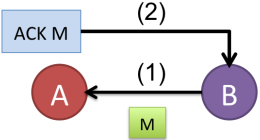

Passive ACK Distribution

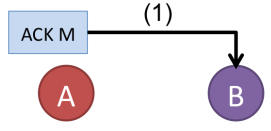

Active ACK Distribution
Figure 1: Passive and Active ACK Distribution Policies

message $m$ to the node that currently holds ACK of $m(A)$. In this case, ACK message will be slowly distributed and copies of $m$ will be slowly discarded from the system. The down point of this policy is the timeliness in distributing ACK message. This is crucial in multi-copy routing scheme since the number of message is growing with time. While the useless message has not been deleted, its replicas will be further generated and forwarded around, consumed more network resources. In active distribution policy, a node holding ACK of any message $\mathrm{m}$ (A) will express its knowledge to its encountering node without any condition on the peer node (B). The extreme case of this active distribution is to express its knowledge, ACK, to every encounter.

There are several reasons why we have chosen an active distribution policy for our design. First, since the communications in DTNs occur opportunistically and often experience disruption, we would like to expediently utilize these connection opportunities by using only one way acknowledgment (Active ACK Distribution) and giving the rest for message transmission. Second, active ACK distribution method would enable us to swiftly discard the copies of delivered messages and prevent them from being regenerated and forwarded to other intermediate nodes. In our design, any node in the system is required to hold a list of known delivered messages ID. However, the size of this list is very small compared to the size of a message. When the contact occurs, encountering nodes will exchange the information in the list. If a message in their buffer matches the ID of delivered message in the list, they will delete corresponding message from their buffers.

To enhance our deletion mechanism, we integrate the knowledge of human mobility patterns into this design.

\subsection{Mobility Patterns and Helper nodes}

As previously mentioned, communications in Delay Tolerant Networks are often facilitated by the mobility of devices. Therefore understanding this mobility patterns can benefit in designing new mechanisms for DTNs. We have integrated the knowledge of human mobility patterns into our deletion mechanism as we introduce the static helper nodes (static H-nodes) based on the idea of "Human Gathering Points". These static H-nodes will play the role in expediting the distribution of ACK messages

Human Gathering Points: The hotspot model is one of the commonly found characteristics of human mobility in various studies $[8,9,10,11]$. This model suggests that there are locations where many people naturally meet in space. This is an essential knowledge in designing the mobile network structure, since it represents strategic locations where a lot of contact opportunities will occur as well as the exchange of 


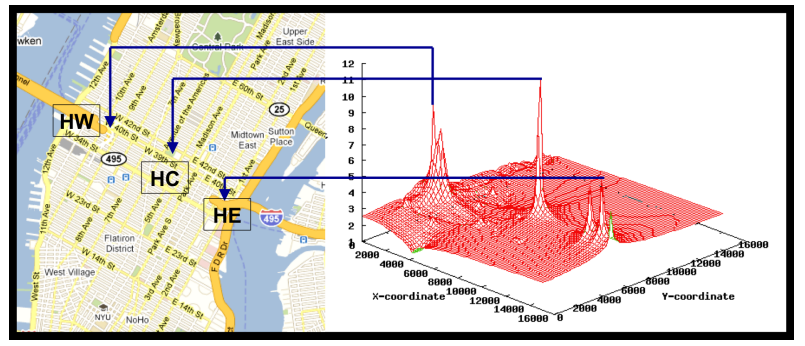

Figure 2: Map of Simulation Area and Location of static H-nodes

messages. We assumed that the popularity of these points will be useful in the distribution of acknowledgment and our deletion mechanism. In the experiment, we deployed the stationary nodes (static H-node) at these locations and study their impacts on our proposed method.

Dataset: The dataset used in our study is the collected GPS traces from Crawdad database [12]. There are 39 GPS traces collected from 10 volunteers in the area of 12 square kilometers in Midtown Manhattan (from 14th Street to 59th Street and from Hudson River to East River). Some of the volunteers are living in Manhattan's vicinity, so their traces are likely to contain long distance travel across from other areas. Their daily means of commuting include subway trains, buses, and walking. We have plotted the frequency of the visits or passes by on each map point (relative $\mathrm{x}, \mathrm{y}$ coordinates), shown in Figure. 2. From the graph we can notice three frequently visited locations. These points are selected as the locations to deploy our static H-nodes. Based on spatial structure of the city, we can also explain the reasons behind these locations' popularity using Space Syntax theory [13]. These three locations include, at the center Herald Square (HC: Hotspot Central), on the east (HE: Hotspot East) and west (HW: Hotspot West) are the entrance/exit to Long Island Expressway and to Lincoln Tunnel respectively. HE and HW have similar characteristics; they serve as port of entry/exit to other areas, which make them popular to a group of nodes that travel across from other areas. While at the center, $\mathrm{HC}$, is a major intersection where total accumulated flow is high, which also infers high integration values in Space Syntax Theory.

\section{PERFORMANCE EVALUATION}

In this section we will explain the evaluation for our proposed method. We first show the simulation environment and then present the results.

\subsection{Simulation Setup}

In order to evaluate our proposed mechanism and the impact of stationary nodes deployed at human gathering points on this mechanism we have simulated our work in the Opportunistic Networking Environment (ONE) simulator [14], which is designed to support DTN nodes characteristics. In addition we also used the OpenJUMP software [15] to tailor our nodes movements according to real collected data traces from the Crawdad dataset. The dataset is the collected traces in Manhattan area, New York City as discussed in Section 2.2. With these tools we were able to simulate our mechanism in more realistic environment.
We simulated three different node-density scenarios on the same terrain, 10-nodes, 20-nodes, and 100-nodes. Table 1 summarizes the simulation parameters. We would like to observe the impact of our method and the effect of human gathering points in the sparse network environments (10 and 20 -nodes scenarios) as well as in the dense network environment (100-nodes scenario). Since the contact opportunity are varied by the density of node. In sparse network the contact opportunities are rare due to the small number of nodes while in dense network, there are frequent contact opportunities. In all scenarios, messages are generated by uniformly selected random sources, towards random destinations. The size of message is also randomly chosen between 500KB-1MB equivalent to the size of large text file or medium size image file. In our simulations, the messages had infinite Time-To-Live (TTL value). Our nodes are all identical, exhibit Bluetooth properties as shown in Table 1. The nodes movement speed is correspondent to human walking speed, $0.5-1.5 \mathrm{~m} / \mathrm{s}$.

To evaluate the impact of stationary nodes deployed at human gathering points, we added one stationary node at each point in respective order according to their frequencies of being visited by distinct nodes. The order and locations are shown in Table 2.

\subsection{Simulation Results}

We use the following performance metrics, namely, delivery probability, overhead, average latency, and average buffer occupancy, to evaluate the performance of our deletion mechanism and the impact of static H-node at Human Gathering Point. The delivery probability metric indicates the successful rate in delivery the message. While overhead and buffer occupancy metrics indicates the resources consumed in delivering the message. Average latency shows the average time required in order to complete the delivering process. These metrics can denote the performance of DTNs message delivery. We implement our algorithm onto three routing protocols which are the representatives of routing protocols in DTNs, Epidemic, Spray and Wait, and ProPHET. Epidemic[3] represents the extreme flooding case, where all messages will be copied to all nodes without any consideration. In contrast, Spray and Wait[4] routing protocol represents the case where every messages are bounded only to limited number of copies, or in another word, controlled flooding. ProPHET[5] routing protocol represents the probabilistic routing protocols, which the number of copies or the nodes carrying the copies, are selected based on history of node encounters and transitivity. This protocol is considered to be adaptive compare to the previous two protocols. The results on each performance metrics are described below.

\subsubsection{Delivery Probability}

Delivery Probability (DP) is the percentage of delivered messages over total unique generated messages.

$$
D P=\frac{\text { DeliveredMessages }}{\text { UniqueGeneratedMessages }}
$$

The delivery probability graphs are shown in Figure. 3, the first column on $\mathrm{x}$-axis (noACK) in all graph represents the default scenario where there is no acknowledgment in the 
Table 1: Scenarios Setting

\begin{tabular}{|l|l|l|l|}
\hline Characteristics & Scenario 1 & Scenario 2 & Scenario 3 \\
\hline Number of Nodes & 10 & 20 & 30 \\
\hline Speed (m/s) & $0.5-1.5$ & $0.5-1.5$ & $0.5-1.5$ \\
\hline Buffer Size (MB) & 20 & 20 & 20 \\
\hline Bandwidth (KB/s) & 250 & 250 & 250 \\
\hline Transmission Range (m) & 10 & 10 & 10 \\
\hline Number of Static H-node & $1-3$ & $1-3$ & $1-3$ \\
\hline Messages Generated Interval (s) & $25-35$ & $25-35$ & $25-35$ \\
\hline
\end{tabular}

Table 2: Static H-node Setting

\begin{tabular}{|c|l|l|}
\hline 250Number of Static H-node & & Location \\
\hline 1 & HC & Herald Square \\
\hline 2 & HW & Entrance/Exit to Lincoln Tunnel \\
\hline 3 & HE & Entrance/Exit to Long Island Expressway \\
\hline
\end{tabular}

system. The second column (ACK) is where the destination node generates the ACK message and distribute to all node without any help from static H-node, our proposed deletion mechanism. Then the rest of the cases involve 1 to 3 static H-nodes at Human Gathering Points $(\mathrm{ACK}+1 \mathrm{hs}$ to $\mathrm{ACK}+3 \mathrm{hs})$. For each routing protocol, Epidemic, ProPHET, Spray and Wait (from left to right), we perform three simulations with different node density as previously described in Section 3 A (10,20,100-nodes).

From Figure.3, we can see that our deletion mechanism can improve the delivery ratio by $10 \%$ in 10 -nodes scenarios, in Epidemic and ProPHET routing protocols. The delivery probabilities also improve by $20 \%$ in 20 -nodes scenario and $50 \%$ in 100 -nodes scenario. The deployment of static H-node at HC (Herald Square) can further improve the delivery performance by $5-15 \%$ in sparse network settings for all routing algorithms. Though, with Spray and Wait routing protocol, our deletion mechanism has smaller improvement. Since Spray and Wait is a flooding-control routing algorithm, it is less likely to be devastated by the message replicas as in other routing protocols. In dense network environment, static H-nodes do not have significant impact due to the high frequency of contact opportunity among moving nodes.

\subsubsection{Overhead}

Overhead is the average resource (storage, bandwidth and energy) consumed per one delivered message. This is the number of replicas that had been relayed in order to achieve one successful delivered message. The layout of Figure. 4, is the same as in Figure 3 described in previous paragraph. In Figure 4, we enlarge the bottom part (below the black horizontal line) of the graphs in order to show the results from sparse network scenarios.

Our proposed mechanism, (ACK, ACK $+1 \mathrm{hs}, \mathrm{ACK}+2 \mathrm{hs}$, $\mathrm{ACK}+3 \mathrm{hs}$ ), shows that it can reduce the resource consumption significantly in Epidemic and ProPHET routing protocols. Since it helps deleting the unnecessary replicas and also preventing nodes from further distributing the replicas of delivered messages. In second column (ACK), where no static H-node is deployed, overhead can be reduced 30-
$50 \%$ in sparse-network scenarios of Epidemic and ProPHET routing protocols. In dense environment it can reduce up to $70 \%$ approximately. However, in Spray and Wait the overhead remains approximately equal to the predefined maximum copies value. Spray and Wait routing protocol allows only $\mathrm{M}$ copies (predefined maximum copies) to be replicated per one message. The static H-nodes at Human Gathering Points causes the overhead to become slightly higher since more replicas are generated to these nodes, however, this can also contribute to higher message delivery probability at the mean time. Single stationary node accounts for $10 \%$ higher in overhead for Epidemic. While in Spray and Wait and ProPHET, one H-node only accounts $3 \%$ higher in overhead.

\subsubsection{Average Latency}

Average Latency is the time interval between sending message by source and receiving its copy by destination.

$$
\text { AverageLatency }=\text { Treceived }- \text { Tsent }
$$

Where Treceived is time when any copy of message $m$ reached at the destination. Tsent is time when message $\mathrm{m}$ is generated at the source.

In Figure 5, we can see that the deletion mechanism increased the average latency in message delivery. However, by deploying the static H-nodes at Human Gathering Points can reduce the average latency in sparse network scenarios for all routing protocols. Epidemic routing protocol case (Figure 5-left), in dense environment, applying our proposed deletion mechanism can vastly reduce the average latency by $35 \%$ compare to the default case where no deletion mechanism is presented. Our mechanism serves as the countermeasure for resource exhaustion, which is likely to occur when using Epidemic routing protocol in dense environment. It allows the network to use the resources more effectively thus contribute to the reduction of average latency in this scenario.

\subsubsection{Average Buffer Occupancy}

Buffer Occupancy (BO) is the percentage of buffer space that occupied by the messages and messages replicas. We 

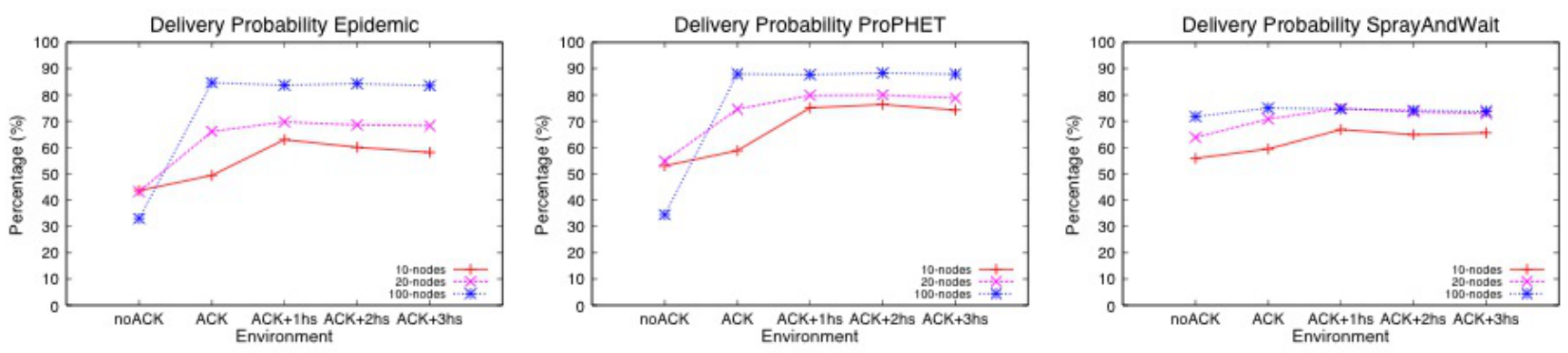

Figure 3: Delivery Probability
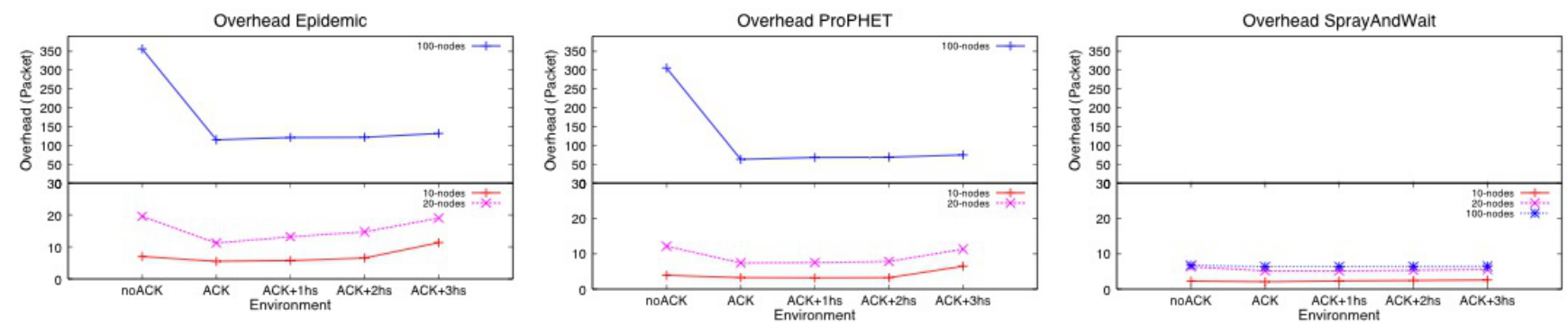

Figure 4: Overhead

have plot the average buffer occupancy (AverageBO) at each simulation time step shown in Figure 6, 7, 8.

$$
\text { Average } B O=\frac{\sum_{i=0}^{n} \text { bufferoccupancy }}{n}
$$

Where $\mathrm{n}$ equals to total number of nodes in the system. When the node's buffer becomes full, the first incoming message in the buffer queue will be dropped by node, in order to make space available for the incoming message. This is done in First-In-First-Out(FIFO) manner. From our perspective, the lower buffer occupancy will prevent the messages from being dropped while it is being forwarded to the destination. Figure 6 , shows the average buffer occupancy in 10-nodes scenario for Epidemic, ProPHET, Spray and Wait protocols. Figure 7 and Figure 8 show average buffer occupancy in 20-nodes and 100- nodes scenarios respectively. The graphs illustrate that our proposed mechanism can reduce the buffer occupancy in all scenarios regardless of applied routing protocols. The default scenario is shown in topmost line which always nearly $100 \%$ occupied and variations of our proposed methods in other lower lines. The impact in dense-environment (100-nodes scenario) is greater than in sparse environment, since ACK messages can spread out more rapidly due to the frequent contact opportunities among nodes. In dense environment we can reduce the buffer occupancy approximately $30-33 \%$ in ProPHET and Spray and Wait. While in sparse network the reduction is about $10-25 \%$.

The static H-nodes deployed at Human Gathering Points can enhance the opportunities of ACK messages distribution as well as opportunities in forwarding messages in sparse network environments. One static $\mathrm{H}$-node at $\mathrm{HC}$ can reduces the buffer occupancy by $10 \%$ in Epidemic and Spray and Wait routing protocols, while reduces $20 \%$ in ProPHET routing protocol. The introduction of static H-nodes at HE and HW decreases the buffer occupancy $10 \%$ more in ProPHET. Since ProPHET is adaptive in nature, where forwarding decision depends on delivery predictability value, so it is able to benefit more from the proposed routing algorithm. It is also likely that our mechanism will have similar impact on other adaptive routing protocols such as potential-based routing protocols.

\section{RELATED WORKS}

As previously mentioned, message and storage management in DTNs was generally integrated with some routing algorithms such as hop-based time-to-live (TTL) in Spray and Wait[4], passive-cure in potential-based entropy adaptive routing (PEAR)[17]. Other works are focusing on buffer management policies[20, 21].

In practical scenario, the selection of routing scheme may bounded under many requirements such as application scenarios. However, some of the routing schemes may not already provide message management function. Therefore the independent message deletion mechanism would be essential in optimizing the performance of these routing scheme. Realizing that network resources, storage, energy, bandwidth are limited, we can foresee many problems. To cope with the problem of buffer overflow/limited storage, one has to consider both preventive measure and procedure to handle the situation once occurs: (i) Preventive measure is the mechanism which is designed to control the buffer space usage by only allowing message with valid status, valid time-to-live 

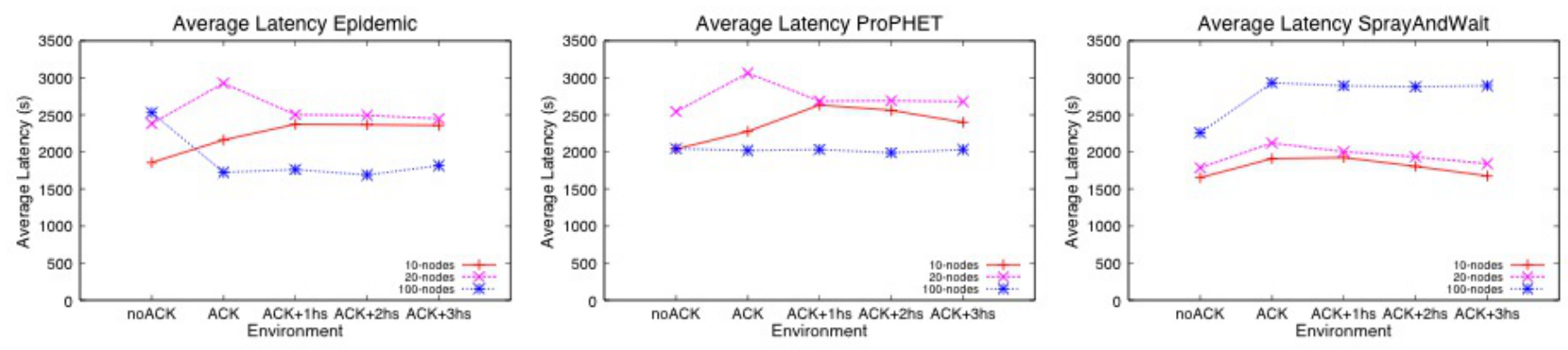

Figure 5: Average Latency
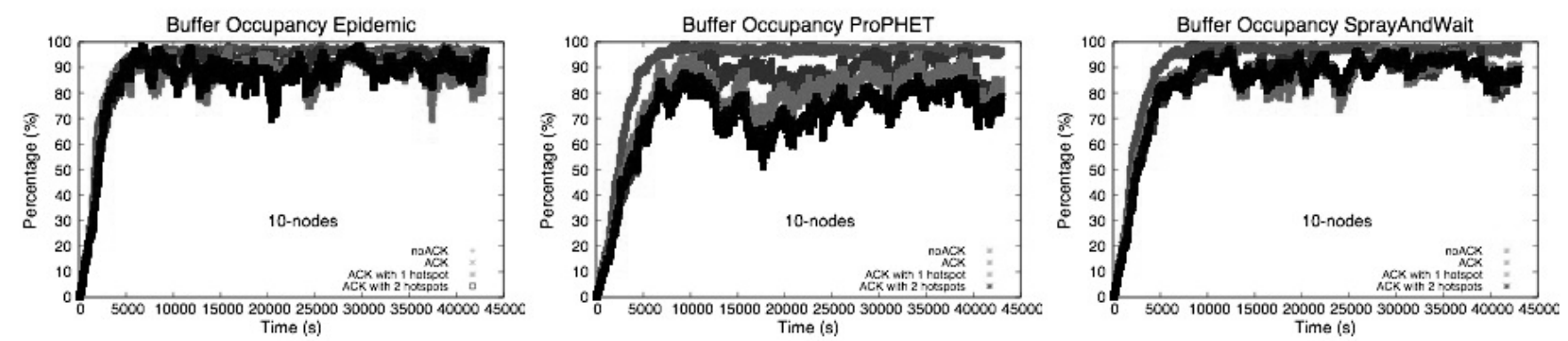

Figure 6: Average Buffer Occupancy in 10-nodes Scenario

(TTL) or has not yet been delivered, in the buffer otherwise it will delete the message. This includes global TTL, local TTL, hop-based TTL, and time-based TTL[18]. Yet, it is difficult to determine an appropriate TTL value. Another method is called anti-packet or passive cure[19], where destination creates the ACK message once it has received the message. Our proposed method falls into this category. However, the method in relaying ACK messages are different from previously proposed algorithm. The previously proposed passive-cure, ACK messages are forwarded in passive approach, as described in Section 2.1. Moreover we take the advantage of nodes' movements and human gathering points to distribute ACK messages more efficiently and effectively. None of the previous works had integrated the human mobility patterns in their studies. The novelty of the design is how to distribute ACK messages to all relevant nodes with minimum resource usage in timely manner. Further optimization of resource use in deleting messages will be considered in our future work.

For (ii) Countermeasure or so called buffer management policies[20, 21] will only take action when node's buffer become full. These policies instruct node in making decision on which packet to be dropped in order to make space available for other messages. For effectiveness of message management, the combination of these methods may be considered as well.

\section{CONCLUSION}

In this paper, we present the independent message deletion mechanism for DTNs multi-copy routing schemes, the enhancement of this method through knowledge of human movements and introduction of static $\mathrm{H}$-node. We evaluate our mechanism by simulations and present the simulation results.

The contributions of this work is two folds; first, the outcomes of effective message management, and second, the benefits of human gathering points on our message deletion mechanism. We have presented the design of an independent message deletion mechanism for DTNs multi-copy routing scheme. By efficiently deleting copies of delivered messages, we can reduce storage consumption and avoid unnecessary bandwidth and energy usage, which in turn enhance the overall performance of DTNs message delivery.

The heuristic knowledge of human movement characteristics has been taken in consideration. We study the impact of "Human Gathering Point", strategic location where people naturally swarm, on our proposed method. The result shows positive impacts in increasing delivery probability and reducing overhead especially in sparse network and for adaptive and potential-based routing scheme. This paper shows that the integration of the knowledge of human movements would be beneficial to future research.

For future works, we would like to extend our proposed method into larger scale. Subsequently, we would consider the corresponding scheme to manage the list of delivered message ID and alternative method for distributing ACK messages such as multicasting scheme. Moreover, we would like to investigate other characteristics of human mobility which might be useful for our design as well as other network protocols. The knowledge of human movements is also provide us the proper simulation environment which similar to the real-world settings. It will be benefit for future 

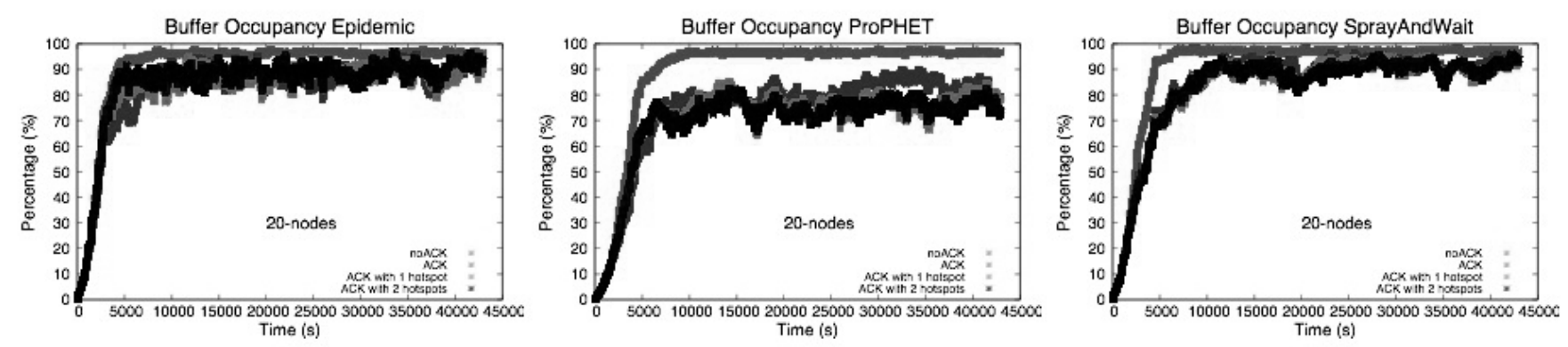

Figure 7: Average Buffer Occupancy in 20-nodes Scenario
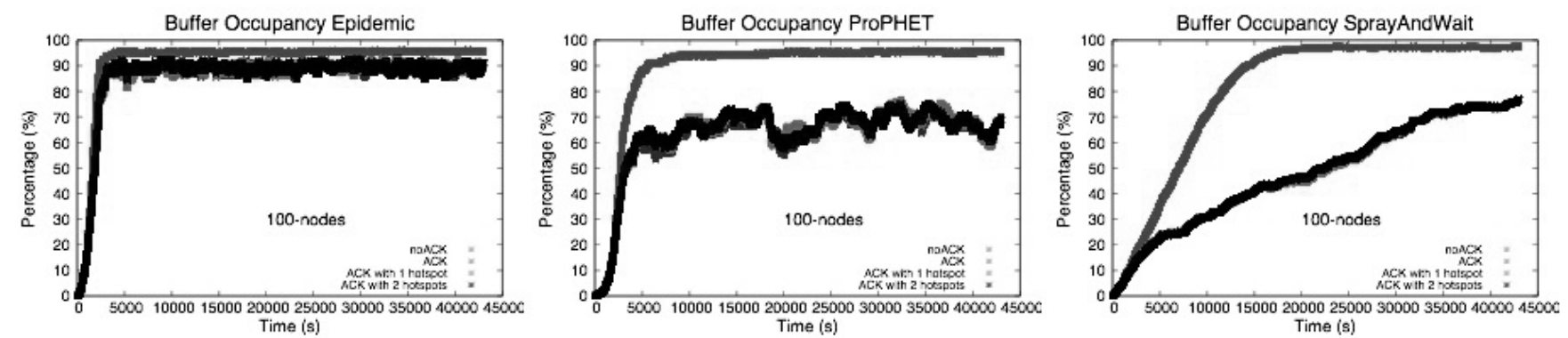

Figure 8: Average Buffer Occupancy in 100-nodes Scenario

deployment.

\section{REFERENCES}

[1] K. Fall, A Delay-Tolerant Network Architecture for Challenged Internets, Proc. ACM SIGCOMM 2003.

[2] S. Jain, K. Fall and R. Patra, Routing in a Delay Tolerant Network, Proc. ACM SIGCOMM 2004.

[3] A. Vahdat and D. Becker, Epidemic routing for partially-connected ad hoc networks, Technical Report CS-200006, Duke University.

[4] T. Spyropoulos, K. Psounis, and C.S. Raghavendra, Spray and Wait: An Efficient Routing Scheme for Intermittently Connected Mobile Networks, Proc. ACM SIGCOMM Workshop on Delay-Tolerant Networking (WDTN) 2005.

[5] A. Lindgren, A. Doria, and O. Schelen, Probabilistic routing in intermittently connected networks, ACM SIGMOBILE Mobile Computing and Communications Review, 7(3): July. 2003.

[6] R.J. D'Souza and J. Jose, Routing Approaches in Delay Tolerant Networks: A Survey, International Journal of Computer Applications (0975-8887) Vol.1, No.17, 2010.

[7] K. Lee, S. Hong, S.J. Kim, I. Rhee, and S. Chong, SLAW: A Mobility Model for Human Walks, Proc. IEEE INFOCOM, 2009.

[8] K. Lee, S. Hong, S.J. Kim, I. Rhee, and S. Chong, Demystifying Levy Walk Patterns in Human Walks, Technical Report, NCSU, http://netsrv.csc.ncsu. edu/export/Demystifying_Levy_Walk_Patterns.pdf, 2008.

[9] V. Borrel, M.D. Amorim, and S. Fdida, A Preferential Attachment Gathering Mobility Model, IEEE
Communications Letters, Vol.9, No.10, October. 2005.

[10] M. Kim, D. Kotz, and S. Kim, Extracting a mobility model from real user traces, Proc. IEEE INFOCOM, 2006.

[11] S. Lim, C. Yu, and C.R. Das, Clustered mobility model for scale-free wireless networks, Proc. IEEE Local Computer Networks (LCN), 2006.

[12] CRAWDAD trace ncsu/mobilitymodels (v. 2009-07-23), Downloaded from http://crawdad.cs.dartmouth.edu /meta.php? name $=$ ncsu/mobilitymodels, December. 2009.

[13] B. Hillier, and L. Vaughan, The city as one thing, Progress in Planning, 67(3). pp.205-230. ISSN 03059006, 2007.

[14] A. Keranan, J. Ott, and T. Karkkainen, The ONE Simulator for DTN Protocol Evaluation, SIMUTOOL, 2009.

[15] OpenJUMP - The free Java based and open source Geographic Information System for the World, http://openjump.org, 2007.

[16] W. Zhao, Y. Chen, M. Ammar, M. Corner, B. Levine, E. Zegura, Capacity Enhancement using Throwboxes in DTNs, IEEE International Conference on Mobile Adhoc and Sensor Systems (MASS), 2006.

[17] H. Ochiai, and H. Esaki, Mobility Entropy and Message Routing in Community-Structured Delay Tolerant Networks AINTEC, November. 2008.

[18] W.H. Yuen, and H. Schulzrinne, Message Replication and Deletion in Delay Tolerant Networks under Hop-based and Time-based TTL Schemes, Columbia University. 
[19] K.A. Harras, and K.C. Almeroth, Transport Layer Issues in Delay Tolerant Mobile Networks, Proc. IFIP Networking, 2006.

[20] D. Passos, H. Bueno, E. Oliveira, and C. Albuquerque, Packet Scheduling and Discard Policies for Diffusion Control in Delay and Disruption Tolerant Networks, Journal of Communication and Information Systems, Vol.23, No.1, pp.12-21, 2008.

[21] A. Krifa, C. Barakat, and T. Spyropoulos, Optimal Buffer Management Policies for Delay Tolerant Networks, Proc. IEEE SECON, 2008. 